\title{
Asymptotically almost periodic solution to a class of Volterra difference equations
}

Wei Long ${ }^{*}$ and Wen-Hai Pan

"Correspondence: hopelw@126.com

College of Mathematics and Information Science, Jiangxi Normal University, Nanchang, Jiangxi 330022, People's Republic of China

\begin{abstract}
This paper is concerned with an asymptotically almost periodic solution to a class of Volterra-type difference equations. We establish a compactness criterion for the sets of asymptotically almost periodic sequences. Then, by using the compactness criterion and Schauder's fixed point theorem, we present an existence theorem for an asymptotically almost periodic solution to the addressed Volterra-type difference equation. Our existence theorem extends and complements a recent result due to (Ding et al. in Electron. J. Qual. Theory Differ. Equ. 6:1-13, 2012).
\end{abstract}

MSC: 39A24; 34K14

Keywords: asymptotically almost periodic; Volterra difference equation

\section{Introduction and preliminaries}

In this paper, we consider the following nonlinear Volterra-type difference equation:

$$
x(n)=\sum_{i=1}^{\lambda}\left[f_{i}(n, x(n)) \cdot \sum_{m \in \mathbb{Z}} a_{i}(n, m) g_{i}(m, x(m))\right], \quad n \in \mathbb{Z},
$$

where $\lambda$ is a fixed positive integer and $f_{i}: \mathbb{Z} \times \mathbb{R} \rightarrow \mathbb{R}, a_{i}: \mathbb{Z} \times \mathbb{Z} \rightarrow \mathbb{R}, g_{i}: \mathbb{Z} \times \mathbb{R} \rightarrow \mathbb{R}$ $(i=1,2, \ldots, \lambda)$ satisfy some conditions recalled in Section 3 .

For the background of discrete Volterra equations, we refer the reader to the well-known monograph [1] by Agarwal. The first motivation for this paper is some recent work on asymptotical periodicity for Volterra-type difference equations in [2-6] by Diblík et al. In fact, asymptotical behavior for Volterra-type difference equations, including periodicity, asymptotical periodicity, etc., has been of great interest for many mathematicians. However, to the best of our knowledge, there is seldom literature available about asymptotically almost periodicity for Equation (1.1). Thus, in this paper, we will investigate this problem. In addition, it is needed to note that compared with asymptotically periodic sequences, in general, it is more difficult to obtain the compactness for a set of asymptotically almost periodic sequences.

On the other hand, in a recent work [7], by using the classical Schauder fixed point theorem, Ding et al. established an interesting existence theorem for the following functional integral equation:

$$
y(t)=e(t, y(\alpha(t)))+g(t, y(\beta(t)))\left[h(t)+\int_{\mathbb{R}} k(t, s) f(s, y(\gamma(s))) d s\right], \quad t \in \mathbb{R} .
$$

\section{Springer}

( 2012 Long and Pan; licensee Springer. This is an Open Access article distributed under the terms of the Creative Commons Attribution License (http://creativecommons.org/licenses/by/2.0), which permits unrestricted use, distribution, and reproduction in any medium, provided the original work is properly cited. 
In fact, the existence of almost periodic type solutions has been an interesting and important topic in the study of qualitative theory of difference equations. We refer the reader to [8-13] and references therein for some recent developments on this topic. Equation (1.1) can be seen as a discrete analogue (but more general) of Equation (1.2). That is another main motivation for this work.

Throughout the rest of this paper, we denote by $\mathbb{Z}\left(\mathbb{Z}^{+}\right)$the set of (nonnegative) integers, by $\mathbb{N}$ the set of positive integers, by $\mathbb{R}\left(\mathbb{R}^{+}\right.$) the set of (nonnegative) real numbers, by $\Omega$ a subset of $\mathbb{R}$, and by $X$ a Banach space.

First, let us recall some notations and basic results of almost periodic type sequences (for more details, see $[11,14,15]$ ).

Definition 1.1 [14] A function $f: \mathbb{Z} \rightarrow X$ is called almost periodic if $\forall \varepsilon, \exists N(\varepsilon) \in \mathbb{N}$ such that among any $N(\varepsilon)$ consecutive integers there exists an integer $p$ with the property that

$$
\|f(k+p)-f(k)\|<\varepsilon, \quad \forall k \in \mathbb{Z} .
$$

Denote by $A P(\mathbb{Z}, X)$ the set of all such functions. Moreover, we denote $A P(\mathbb{Z}, \mathbb{R})$ by $A P(\mathbb{Z})$ for convenience.

Lemma 1.2 [14, Theorem 1.26] A necessary and sufficient condition for the sequence $f: \mathbb{Z} \rightarrow \mathbb{R}$ to be almost periodic is that for any integer sequence $\left\{n_{k}^{\prime}\right\}$, one can extract a subsequence $\left\{n_{k}\right\}$ such that $\left\{f\left(n+n_{k}\right)\right\}$ converges uniformly with respect to $n \in \mathbb{Z}$.

Remark 1.3 Let $f, g \in A P(\mathbb{Z})$. By Lemma 1.2, it is not difficult to show that $\forall \varepsilon, \exists N(\varepsilon) \in \mathbb{N}$ such that among any $N(\varepsilon)$ consecutive integers there exists a common integer $p$ with the property that

$$
|f(k+p)-f(k)|<\varepsilon \quad \text { and } \quad|g(k+p)-g(k)|<\varepsilon
$$

for all $k \in \mathbb{Z}$.

Next, we denote by $C_{0}(\mathbb{Z}, X)$ the space of all the functions $f: \mathbb{Z} \rightarrow X$ such that $\lim _{|n| \rightarrow \infty}\|f(n)\|=0$.

Definition 1.4 A function $f: \mathbb{Z} \rightarrow X$ is called asymptotically almost periodic if it admits a decomposition $f=g+h$, where $g \in A P(\mathbb{Z}, X)$ and $h \in C_{0}(\mathbb{Z}, X)$. Denote by $A A P(\mathbb{Z}, X)$ the set of all such functions. Moreover, we denote $A A P(\mathbb{Z}, \mathbb{R})$ by $A A P(\mathbb{Z})$ for convenience.

Definition 1.5 Let $\Omega \subset \mathbb{R}$ and $f$ be a function from $\mathbb{Z} \times \Omega$ to $\mathbb{R}$ such that $f(n, \cdot)$ is continuous for each $n \in \mathbb{Z}$. Then $\mathrm{f}$ is called almost periodic in $\mathrm{n} \in \mathbb{Z}$ uniformly for $\omega \in \Omega$ if for every $\varepsilon>0$ and every compact $\Sigma \subset \Omega$, there corresponds an integer $N_{\varepsilon}(\Sigma)>0$ such that among $N_{\varepsilon}(\Sigma)$ consecutive integers there exists an integer $p$ with the property that

$$
|f(k+p, \omega)-f(k, \omega)|<\varepsilon
$$

for all $k \in \mathbb{Z}$ and $\omega \in \Sigma$. Denote by $A P(\mathbb{Z} \times \Omega)$ the set of all such functions. 
Similarly, for each subset $\Omega \subset \mathbb{R}$, we denote by $C_{0}(\mathbb{Z} \times \Omega)$ the space of all the functions $f$ : $\mathbb{Z} \times \Omega \rightarrow \mathbb{R}$ such that $f(n, \cdot)$ is continuous for each $n \in \mathbb{Z}$, and $\lim _{|n| \rightarrow \infty} f(n, x)=0$ uniformly for $x$ in any compact subset of $\Omega$.

Definition 1.6 A function $f: \mathbb{Z} \times \Omega \rightarrow \mathbb{R}$ is called asymptotically almost periodic in $n$ uniformly for $x \in \Omega$ if it admits a decomposition $f=g+h$, where $g \in A P(\mathbb{Z} \times \Omega)$ and $h \in C_{0}(\mathbb{Z} \times \Omega)$. Denote by $A A P(\mathbb{Z} \times \Omega)$ the set of all such functions.

Lemma 1.7 Let $E \in\{A P(\mathbb{Z}, X), A A P(\mathbb{Z}, X)\}$. Then the following hold true:

(a) $f \in E$ implies that $f$ is bounded.

(b) $f, g \in E$ implies that $f+g \in E$. Moreover, $f \cdot g \in E$ if $X=\mathbb{R}$.

(c) $E$ is a Banach space equipped with the supremum norm.

Proof The proof is similar to that of the continuous case $(c f .[14,15])$. So, we omit the details.

\section{A compactness criterion}

The following theorem is a well-known result for the continuous case (see, e.g., [16, p.24, Theorem 2.5]). Here, we give a discrete version.

Theorem 2.1 Let $f$ be a function from $\mathbb{Z}$ to $\mathbb{R}$. Then $f \in A A P(\mathbb{Z})$ if and only if $\forall \varepsilon$, $\exists M(\varepsilon), N(\varepsilon) \in \mathbb{N}$ such that among any $N(\varepsilon)$ consecutive integers there exists an integer $p$ with the property that

$$
|f(k+p)-f(k)|<\varepsilon
$$

for all $k \in \mathbb{Z}$ with $|k| \geq M(\varepsilon)$ and $|k+p| \geq M(\varepsilon)$.

Proof We first show the 'only if' part. Let $f \in A A P(\mathbb{Z})$. Then there exist $g \in A P(\mathbb{Z})$ and $h \in C_{0}(\mathbb{Z}, \mathbb{R})$ such that $f=g+h$. By $g \in A P(\mathbb{Z})$, for each $\varepsilon>0, \exists N(\varepsilon) \in \mathbb{N}$ such that among any $N(\varepsilon)$ consecutive integers there exists an integer $p$ with the property that

$$
|g(k+p)-g(k)|<\frac{\varepsilon}{3}, \quad \forall k \in \mathbb{Z}
$$

In addition, since $h \in C_{0}(\mathbb{Z}, \mathbb{R})$, for the above $\varepsilon>0$, there exists $M(\varepsilon) \in \mathbb{N}$ such that $|h(k)|<$ $\frac{\varepsilon}{3}$ for all $k \in \mathbb{Z}$ with $|k| \geq M(\varepsilon)$. Thus, we have

$$
|f(k+p)-f(k)| \leq|g(k+p)-g(k)|+|h(k+p)|+|h(k)|<\varepsilon
$$

for all $k \in \mathbb{Z}$ with $|k| \geq M(\varepsilon)$ and $|k+p| \geq M(\varepsilon)$.

Next, let us prove the 'if' part. First, let us show that $f$ is bounded. Letting $\varepsilon=1$, there exists $M(1), N(1) \in \mathbb{N}$ such that among any $N(1)$ consecutive integers there exists an integer $p$ with the property that

$$
|f(k+p)-f(k)|<1
$$


for all $k \in \mathbb{Z}$ with $|k| \geq M(1)$ and $|k+p| \geq M(1)$. Then, for each $k \in \mathbb{Z}$ with $|k| \geq M(1)$, there exists $p_{k} \in[M(1)-k, M(1)+N(1)-k] \cap \mathbb{Z}$ such that

$$
\left|f\left(k+p_{k}\right)-f(k)\right|<1
$$

Noting that $k+p_{k} \in[M(1), M(1)+N(1)]$, we get

$$
|f(k)| \leq\left|f\left(k+p_{k}\right)\right|+1 \leq \max _{k \in[M(1), M(1)+N(1)]}|f(k)|+1
$$

for all $k \in \mathbb{Z}$ with $|k| \geq M(1)$. Thus,

$$
\sup _{k \in \mathbb{Z}}|f(k)| \leq \max _{k \in[-M(1), M(1)+N(1)]}|f(k)|+1<+\infty .
$$

Now, let us show that $f \in A A P(\mathbb{Z})$. We divide the remaining proof into three steps.

Step 1. Since $f$ is bounded, we can choose a sequence $\left\{s_{n}\right\} \subset \mathbb{N}$ such that $\lim _{n \rightarrow+\infty} s_{n}=$ $+\infty$ and $\lim _{n \rightarrow+\infty} f\left(k+s_{n}\right)$ exists for each $k \in \mathbb{Z}$. Let

$$
\bar{g}(k)=\lim _{n \rightarrow+\infty} f\left(k+s_{n}\right), \quad k \in \mathbb{Z} .
$$

For each $\varepsilon>0$, among any $N(\varepsilon)$ consecutive integers there exists an integer $p$ with the property that

$$
|f(k+p)-f(k)|<\varepsilon
$$

for all $k \in \mathbb{Z}$ with $|k| \geq M(\varepsilon)$ and $|k+p| \geq M(\varepsilon)$. Then, for each fixed $k \in \mathbb{Z}$, we have

$$
\left|f\left(k+s_{n}+p\right)-f\left(k+s_{n}\right)\right|<\varepsilon
$$

for sufficiently large $n$, which yields that

$$
|\bar{g}(k+p)-\bar{g}(k)| \leq \varepsilon .
$$

Thus, $\bar{g} \in A P(\mathbb{Z})$.

Step 2. Now fix $\varepsilon>0$. Then, for each $n \in \mathbb{N}$, there exists $t_{n} \in\left[s_{n}-N(\varepsilon), s_{n}\right] \cap \mathbb{Z}$ such that

$$
\left|f\left(k+t_{n}\right)-f(k)\right|<\varepsilon
$$

for all $k \in \mathbb{Z}$ with $|k| \geq M(\varepsilon)$ and $\left|k+t_{n}\right| \geq M(\varepsilon)$. Let $r_{n}=s_{n}-t_{n}$. Then $r_{n} \in\{0,1,2, \ldots, N(\varepsilon)\}$, which means that there exist a subsequence $\left\{r_{n}^{\prime}\right\} \subset\left\{r_{n}\right\}$ and $r(\varepsilon) \in\{0,1,2, \ldots, N(\varepsilon)\}$ such that

$$
r_{n}^{\prime} \equiv r(\varepsilon)
$$

Thus, for all $k \in \mathbb{Z}$ with $|k| \geq M(\varepsilon)$, we have

$$
|f(k)-\bar{g}(k-r(\varepsilon))|=\left|f(k)-f\left(k+t_{n}^{\prime}\right)\right|+\left|f\left(k-r(\varepsilon)+s_{n}^{\prime}\right)-\bar{g}(k-r(\varepsilon))\right| .
$$


Combining this with (2.1), $\lim _{n \rightarrow+\infty} t_{n}^{\prime}=+\infty$, and

$$
\bar{g}(k)=\lim _{n \rightarrow+\infty} f\left(k+s_{n}\right), \quad \forall k \in \mathbb{Z}
$$

we conclude

$$
|f(k)-\bar{g}(k-r(\varepsilon))| \leq \varepsilon
$$

for all $k \in \mathbb{Z}$ with $|k| \geq M(\varepsilon)$.

Step 3. By Step 2, we know that for each $\varepsilon>0$, there exists $r(\varepsilon) \in\{0,1,2, \ldots, N(\varepsilon)\}$ such that

$$
|f(k)-\bar{g}(k-r(\varepsilon))| \leq \varepsilon
$$

for all $k \in \mathbb{Z}$ with $|k| \geq M(\varepsilon)$. Taking $\varepsilon=1,1 / 2, \ldots$, we get a sequence $\{r(1 / m)\}$. On the other hand, it follows from Step 1 that $\bar{g} \in A P(\mathbb{Z})$. Thus, going to a subsequence, if necessary, we may assume that $\bar{g}(\cdot-r(1 / m))$ is uniformly convergent on $\mathbb{Z}$. Let

$$
g(k)=\lim _{m \rightarrow+\infty} \bar{g}(k-r(1 / m)), \quad k \in \mathbb{Z} .
$$

Then $g \in A P(\mathbb{Z})$. In addition, noting that

$$
\begin{aligned}
|f(k)-g(k)| & \leq|f(k)-\bar{g}(k-r(1 / m))|+|\bar{g}(k-r(1 / m))-g(k)| \\
& \leq \frac{1}{m}+|\bar{g}(k-r(1 / m))-g(k)|
\end{aligned}
$$

for all $k \in \mathbb{Z}$ with $|k| \geq M(1 / m)$, we know that $f-g \in C_{0}(\mathbb{Z})$. This completes the proof.

Definition 2.2 $F \subseteq A A P(\mathbb{Z})$ is said to be equi-asymptotically almost periodic if for each $\varepsilon>0$, there exist $M(\varepsilon), N(\varepsilon) \in \mathbb{N}$ such that among any $N(\varepsilon)$ consecutive integers there exists an integer $p$ with the property that

$$
\sup _{f \in F}|f(k+p)-f(k)|<\varepsilon
$$

for all $k \in \mathbb{Z}$ with $|k| \geq M(\varepsilon)$ and $|k+p| \geq M(\varepsilon)$.

Theorem 2.3 Let $F \subseteq A A P(\mathbb{Z})$. Then $F$ is precompact in $A A P(\mathbb{Z})$ if and only if the following two conditions hold:

(i) for each $k \in \mathbb{Z},\{f(k): f \in F\}$ is bounded;

(ii) $F$ is equi-asymptotically almost periodic.

Proof 'only if' part

Let $F \subset A A P(\mathbb{Z})$ be precompact. Then $F$ is bounded in $A A P(\mathbb{Z})$. So, (i) obviously holds. In addition, $\forall \varepsilon>0$, there exists $N \in \mathbb{N}$ and $f_{1}, f_{2}, \ldots, f_{N} \in F$ such that

$$
F \subset \bigcup_{i=1}^{N} B\left(f_{i}, \varepsilon\right) .
$$


By Remark 1.3 , we can get that $\left\{f_{1}, f_{2}, \ldots, f_{N}\right\}$ is equi-asymptotically almost periodic. Combing this with (2.2), we can show that $F$ is equi-asymptotically almost periodic, i.e., (ii) holds. 'if part'

Let $\left\{f_{n}\right\} \subset F$. Since $\left\{f_{n}(k)\right\}$ is bounded for each $k \in \mathbb{Z}$, we can assume that (if necessary going to a subsequence) $\left\{f_{n}(k)\right\}$ is convergent for each $k \in \mathbb{Z}$. On the other hand, since $F$ is equi-asymptotically almost periodic, for each $\varepsilon>0$, there exist $M(\varepsilon), N(\varepsilon) \in \mathbb{N}$ such that among any $N(\varepsilon)$ consecutive integers there exists an integer $p$ with the property that

$$
\sup _{n \in \mathbb{Z}}\left|f_{n}(k+p)-f_{n}(k)\right|<\varepsilon / 3
$$

for all $k \in \mathbb{Z}$ with $|k| \geq M(\varepsilon)$ and $|k+p| \geq M(\varepsilon)$. For the above $\varepsilon>0$, there exists a positive integer $K$ such that for all $n, m>K$, the following hold:

$$
\left|f_{n}(k)-f_{m}(k)\right|<\varepsilon / 3, \quad k \in[-M(\varepsilon), M(\varepsilon)+N(\varepsilon)] \cap \mathbb{Z} .
$$

For all $k \in \mathbb{Z}$ with $|k| \geq M(\varepsilon)$, taking $p \in[-k+M(\varepsilon),-k+M(\varepsilon)+N(\varepsilon)] \cap \mathbb{Z}$, by (2.3) and (2.4), we get

$$
\begin{aligned}
\left|f_{n}(k)-f_{m}(k)\right| & \leq\left|f_{n}(k)-f_{n}(k+p)\right|+\left|f_{n}(k+p)-f_{m}(k+p)\right|+\left|f_{m}(k+p)-f_{m}(k)\right| \\
& <\varepsilon / 3+\varepsilon / 3+\varepsilon / 3=\varepsilon, \quad n, m>K ;
\end{aligned}
$$

also, for all $k \in \mathbb{Z}$ with $|k|<M(\varepsilon)$, by (2.4), we have

$$
\left|f_{n}(k)-f_{m}(k)\right|<\varepsilon / 3<\varepsilon, \quad n, m>K \text {. }
$$

Thus, we get

$$
\sup _{k \in \mathbb{Z}}\left|f_{n}(k)-f_{m}(k)\right| \leq \varepsilon, \quad n, m>K
$$

which means that $\left\{f_{n}(k)\right\}$ is uniformly convergent on $\mathbb{Z}$, i.e., $\left\{f_{n}\right\}$ is convergent in $A A P(\mathbb{Z})$. So, $F$ is precompact in $A A P(\mathbb{Z})$.

\section{Application to Volterra difference equations}

In this section, we discuss the existence of an asymptotically almost periodic solution to Volterra difference equation (1.1). Throughout the rest of this paper, $p, q \geq 1$ are two fixed real numbers and

$$
\frac{1}{p}+\frac{1}{q}=1
$$

In addition, we denote by $l^{p}(\mathbb{Z})\left(\right.$ resp. $\left.l^{q}(\mathbb{Z})\right)$ the space of all the functions $f: \mathbb{Z} \rightarrow \mathbb{R}$ satisfying

$$
\|f\|_{p}:=\left(\sum_{k \in \mathbb{Z}}|f(k)|^{p}\right)^{1 / p}<+\infty \quad\left(\text { resp. }\|f\|_{q}:=\left(\sum_{k \in \mathbb{Z}}|f(k)|^{q}\right)^{1 / q}<+\infty\right) .
$$

For convenience, we first list some assumptions. 
(H1) For each $i \in\{1,2, \ldots, \lambda\}, f_{i}(\cdot, x) \in A A P(\mathbb{Z})$ for any fixed $x \in \mathbb{R}$, and there exists a constant $L_{i} \geq 0$ such that

$$
\left|f_{i}(k, x)-f_{i}(k, y)\right| \leq L_{i}|x-y|, \quad \forall k \in \mathbb{Z}, \forall x, y \in \mathbb{R} .
$$

(H2) For each $i \in\{1,2, \ldots, \lambda\}, g_{i}(k, \cdot)$ is continuous for each $k \in \mathbb{Z}$, and for each $r>0$, there exists a sequence $\left\{\mu_{i}^{r}\right\} \subset l^{p}(\mathbb{Z})$ such that

$$
\left|g_{i}(k, x)\right| \leq \mu_{i}^{r}(k), \quad|x| \leq r, k \in \mathbb{Z}
$$

(H3) For each $i \in\{1,2, \ldots, \lambda\}, \widetilde{a}_{i} \in A A P\left(\mathbb{Z}, l^{q}(\mathbb{Z})\right)$, where $\left[\widetilde{a}_{i}(k)\right](l)=a_{i}(k, l), \forall k, l \in \mathbb{Z}$.

(H4) There exists a constant $M>0$ such that

$$
\begin{aligned}
& \qquad \sum_{i=1}^{\lambda} \alpha_{i} L_{i}\left\|\mu_{i}^{M}\right\|_{p}<1, \\
& \text { where } \alpha_{i}=\sup _{n \in \mathbb{Z}}\left\|\widetilde{a}_{i}(n)\right\|_{q} ; \text { and } \\
& \qquad \sum_{i=1}^{\lambda}\left[\sup _{n \in \mathbb{Z},|x| \leq K}\left|f_{i}(n, x)\right| \cdot \alpha_{i} \cdot\left\|\mu_{i}^{M}\right\|_{p}\right]<K, \quad \forall K>M,
\end{aligned}
$$

Theorem 3.1 Assume that (H1)-(H4) hold. Then Equation (1.1) has an asymptotically almost periodic solution.

Proof We denote

$$
\begin{aligned}
& \left(A_{i} x\right)(n)=f_{i}(n, x(n)), \quad n \in \mathbb{Z}, x \in A A P(\mathbb{Z}), i=1,2, \ldots, \lambda ; \\
& \left(B_{i} x\right)(n)=\sum_{m \in \mathbb{Z}} a_{i}(n, m) g_{i}(m, x(m)), \quad n \in \mathbb{Z}, x \in A A P(\mathbb{Z}), i=1,2, \ldots, \lambda ;
\end{aligned}
$$

and

$$
(\mathcal{M} x)(n)=\sum_{i=1}^{\lambda}\left(A_{i} x\right)(n) \cdot\left(B_{i} x\right)(n), \quad n \in \mathbb{Z}, x \in A A P(\mathbb{Z}) .
$$

It suffices to prove that $\mathcal{M}$ has a fixed point in $A A P(\mathbb{Z})$. We give the proof in three steps.

Step 1. $A_{i}$ and $B_{i}$ both map $A A P(\mathbb{Z})$ into $A A P(\mathbb{Z}), i=1,2, \ldots, \lambda$.

Since $f_{i}$ is Lipschitz, by Remark 1.3 , we can first show that for each compact subset $K \subset \mathbb{R}$ and each $i \in\{1,2, \ldots, \lambda\},\left\{f_{i}(\cdot, x): x \in K\right\}$ is equi-asymptotically almost periodic. Then it is easy to show that $A_{i} x \in A A P(\mathbb{Z})$ for each $x \in A A P(\mathbb{Z})$.

Since $\widetilde{a}_{i} \in A A P\left(\mathbb{Z}, l^{q}(\mathbb{Z})\right)$, there exist $b_{i} \in A P\left(\mathbb{Z}, l^{q}(\mathbb{Z})\right)$ and $c_{i} \in C_{0}\left(\mathbb{Z}, l^{q}(\mathbb{Z})\right)$ such that $\widetilde{a}_{i}=$ $b_{i}+c_{i}$. For each $x \in A A P(\mathbb{Z})$, noting that for $n, p \in \mathbb{Z}$,

$$
\begin{aligned}
\left(B_{i} x\right)(n) & =\sum_{m \in \mathbb{Z}} a_{i}(n, m) g_{i}(m, x(m)) \\
& =\sum_{m \in \mathbb{Z}}\left[b_{i}(n)\right](m) g_{i}(m, x(m))+\sum_{m \in \mathbb{Z}}\left[c_{i}(n)\right](m) g_{i}(m, x(m)),
\end{aligned}
$$




$$
\begin{aligned}
& \left|\sum_{m \in \mathbb{Z}}\left[b_{i}(n+p)\right](m) g_{i}(m, x(m))-\sum_{m \in \mathbb{Z}}\left[b_{i}(n)\right](m) g_{i}(m, x(m))\right| \\
& \quad \leq\left\|b_{i}(n+p)-b_{i}(n)\right\|_{q} \cdot\left\|\mu_{i}^{\|x\|}\right\|_{p}
\end{aligned}
$$

and

$$
\left|\sum_{m \in \mathbb{Z}}\left[c_{i}(n)\right](m) g_{i}(m, x(m))\right| \leq\left\|c_{i}(n)\right\|_{q} \cdot\left\|\mu_{i}^{\|x\|}\right\|_{p}
$$

we know that $B_{i} x \in A A P(\mathbb{Z})$.

Step 2. For each $y \in A A P(\mathbb{Z})$ with $\|y\| \leq M$, there exists a unique $x_{y} \in A A P(\mathbb{Z})$ such that

$$
x_{y}=\sum_{i=1}^{\lambda} A_{i} x_{y} \cdot B_{i} y
$$

Let

$$
\left(\mathcal{Y}_{x}\right)(n)=\sum_{i=1}^{\lambda}\left(A_{i} x\right)(n) \cdot\left(B_{i} y\right)(n), \quad n \in \mathbb{Z}, x \in A A P(\mathbb{Z}) .
$$

Then, by Step $1, \mathcal{Y}$ maps $A A P(\mathbb{Z})$ into $A A P(\mathbb{Z})$. For all $x_{1}, x_{2} \in A A P(\mathbb{Z})$ and $n \in \mathbb{Z}$, we have

$$
\begin{aligned}
& \left|\left(\mathcal{Y} x_{1}\right)(n)-\left(\mathcal{Y} x_{2}\right)(n)\right| \\
& \leq \sum_{i=1}^{\lambda}\left|\left(A_{i} x_{1}\right)(n)-\left(A_{i} x_{2}\right)(n)\right| \cdot\left|\left(B_{i} y\right)(n)\right| \\
& =\sum_{i=1}^{\lambda}\left|f_{i}\left(n, x_{1}(n)\right)-f_{i}\left(n, x_{2}(n)\right)\right| \cdot\left|\left(B_{i} y\right)(n)\right| \\
& \leq \sum_{i=1}^{\lambda} L_{i}\left|x_{1}(n)-x_{2}(n)\right| \cdot\left|\sum_{m \in \mathbb{Z}} a_{i}(n, m) g_{i}(m, y(m))\right| \\
& \leq \sum_{i=1}^{\lambda} L_{i}\left\|x_{1}-x_{2}\right\| \cdot\left|\sum_{m \in \mathbb{Z}} a_{i}(n, m) g_{i}(m, y(m))\right| \\
& \leq \sum_{i=1}^{\lambda} L_{i}\left\|x_{1}-x_{2}\right\| \cdot \sum_{m \in \mathbb{Z}}\left|\left[\tilde{a}_{i}(n)\right](m)\right| \cdot \mu_{i}^{M}(m) \\
& \leq \sum_{i=1}^{\lambda} L_{i}\left\|x_{1}-x_{2}\right\| \cdot\left\|\tilde{a}_{i}(n)\right\|_{q} \cdot\left\|\mu_{i}^{M}\right\|_{p} \\
& \leq\left(\sum_{i=1}^{\lambda} \alpha_{i} L_{i}\left\|\mu_{i}^{M}\right\| \|_{p}\right)\left\|x_{1}-x_{2}\right\|,
\end{aligned}
$$

which yields that

$$
\left\|\mathcal{Y} x_{1}-\mathcal{Y} x_{2}\right\| \leq\left(\sum_{i=1}^{\lambda} \alpha_{i} L_{i}\left\|\mu_{i}^{M}\right\|_{p}\right)\left\|x_{1}-x_{2}\right\| .
$$


Noting that $\sum_{i=1}^{\lambda} \alpha_{i} L_{i}\left\|\mu_{i}^{M}\right\|_{p}<1, \mathcal{Y}$ has a unique fixed point $x_{y}$ in $A A P(\mathbb{Z})$.

Step 3. $\mathcal{M}$ has a fixed point in $A A P(\mathbb{Z})$.

Let $E=\{y \in A A P(\mathbb{Z}):\|y\| \leq M\}$ and

$$
\mathcal{N} y=x_{y}, \quad y \in E,
$$

where $x_{y}$ is the unique fixed point of $\mathcal{Y}$ (see Step 2).

We claim that $\mathcal{N}(E) \subset E$. In fact, if there exists $y_{0} \in E$ such that $\left\|\mathcal{N} y_{0}\right\|>M$, then by (H4), we have

$$
\begin{aligned}
\left\|\mathcal{N} y_{0}\right\| & =\left\|x_{y_{0}}\right\| \\
& =\sup _{n \in \mathbb{Z}}\left|\sum_{i=1}^{\lambda}\left(A_{i} x_{y_{0}}\right)(n) \cdot\left(B_{i} y_{0}\right)(n)\right| \\
& \leq \sup _{n \in \mathbb{Z}}\left(\sum_{i=1}^{\lambda}\left|f_{i}\left(n, x_{y_{0}}(n)\right)\right| \cdot\left|\sum_{m \in \mathbb{Z}} a_{i}(n, m) g_{i}\left(m, y_{0}(m)\right)\right|\right) \\
& \leq \sup _{n \in \mathbb{Z}}\left(\sum_{i=1}^{\lambda}\left|f_{i}\left(n, x_{y_{0}}(n)\right)\right| \cdot \alpha_{i} \cdot\left\|\mu_{i}^{M}\right\|_{p}\right) \\
& \leq \sum_{i=1}^{\lambda}\left[\sup _{n \in \mathbb{Z},|x| \leq\left\|\mathcal{N} y_{0}\right\|}\left|f_{i}(n, x)\right| \cdot \alpha_{i} \cdot\left\|\mu_{i}^{M}\right\|_{p}\right] \\
& <\left\|\mathcal{N} y_{0}\right\|,
\end{aligned}
$$

which is a contradiction.

Next, let us show that $\mathcal{N}: E \rightarrow E$ is continuous. For all $y_{1}, y_{2} \in E$, we have

$$
\begin{aligned}
\left\|\mathcal{N} y_{1}-\mathcal{N} y_{2}\right\|= & \left\|x_{y_{1}}-x_{y_{2}}\right\| \\
= & \left\|\sum_{i=1}^{\lambda} A_{i} x_{y_{1}} \cdot B_{i} y_{1}-\sum_{i=1}^{\lambda} A_{i} x_{y_{2}} \cdot B_{i} y_{2}\right\| \\
\leq & \sum_{i=1}^{\lambda}\left\|A_{i} x_{y_{1}} \cdot B_{i} y_{1}-A_{i} x_{y_{2}} \cdot B_{i} y_{1}+A_{i} x_{y_{2}} \cdot B_{i} y_{1}-A_{i} x_{y_{2}} \cdot B_{i} y_{2}\right\| \\
\leq & \left(\sum_{i=1}^{\lambda} \alpha_{i} L_{i}\left\|\mu_{i}^{M}\right\|_{p}\right)\left\|x_{y_{1}}-x_{y_{2}}\right\| \\
& +\sum_{i=1}^{\lambda}\left(M L_{i}+\sup _{n \in \mathbb{Z}}\left|f_{i}(n, 0)\right|\right) \cdot\left\|B_{i} y_{1}-B_{i} y_{2}\right\|,
\end{aligned}
$$

which gives that

$$
\left\|\mathcal{N} y_{1}-\mathcal{N} y_{2}\right\| \leq \sum_{i=1}^{\lambda} \beta_{i} \cdot\left\|B_{i} y_{1}-B_{i} y_{2}\right\|,
$$


where

$$
\beta_{i}:=\frac{M L_{i}+\sup _{n \in \mathbb{Z}}\left|f_{i}(n, 0)\right|}{1-\left(\sum_{i=1}^{\lambda} \alpha_{i} L_{i}\left\|\mu_{i}^{M}\right\|_{p}\right)}, \quad i=1,2, \ldots, \lambda .
$$

Letting $y_{k} \rightarrow y$ in $E$, by (3.1), we have

$$
\begin{aligned}
\left\|\mathcal{N} y_{k}-\mathcal{N} y\right\| & \leq \sum_{i=1}^{\lambda} \beta_{i} \cdot\left\|B_{i} y_{k}-B_{i} y\right\| \\
& \leq \sum_{i=1}^{\lambda} \beta_{i} \cdot \sup _{n \in \mathbb{Z}}\left(\sum_{m \in \mathbb{Z}}\left|a_{i}(n, m)\right| \cdot\left|g_{i}\left(m, y_{k}(m)\right)-g_{i}(m, y(m))\right|\right) \\
& \leq \sum_{i=1}^{\lambda} \beta_{i} \cdot \sup _{n \in \mathbb{Z}}\left(\left\|\tilde{a}_{i}(n)\right\|_{q} \cdot\left\|g_{i}\left(\cdot, y_{k}(\cdot)\right)-g_{i}(\cdot, y(\cdot))\right\|_{p}\right) \\
& \leq \sum_{i=1}^{\lambda} \alpha_{i} \beta_{i} \cdot\left\|g_{i}\left(\cdot, y_{k}(\cdot)\right)-g_{i}(\cdot, y(\cdot))\right\|_{p} .
\end{aligned}
$$

For each $i=1,2, \ldots, \lambda$, noting that

$$
\left|g_{i}\left(m, y_{k}(m)\right)-g_{i}(m, y(m))\right| \leq 2 \mu_{i}^{M}(m), \quad m \in \mathbb{Z}
$$

$g_{i}(m, \cdot)$ is continuous for each $m \in \mathbb{Z}$, and $y_{k}(m) \rightarrow y(m)$ for each $m \in \mathbb{Z}$, we conclude that

$$
\left\|g_{i}\left(\cdot, y_{k}(\cdot)\right)-g_{i}(\cdot, y(\cdot))\right\|_{p} \rightarrow 0 .
$$

Combining this with (3.2), we know that $\mathcal{N} y_{k} \rightarrow \mathcal{N} y$. So $\mathcal{N}: E \rightarrow E$ is continuous.

Now, let us show that $\mathcal{N}(E)$ is precompact in $A A P(\mathbb{Z})$. In order to show that, we first prove each $B_{i}(E)$ is precompact in $A A P(\mathbb{Z})$. By a direct calculation, we can get

$$
\left|\left(B_{i} y\right)(n)\right| \leq \alpha_{i} \cdot\left\|\mu_{i}^{M}\right\|_{p}, \quad i=1,2, \ldots, \lambda,
$$

for all $y \in E$ and $n \in \mathbb{Z}$. In addition, for all $n_{1}, n_{2} \in \mathbb{Z}$ and $y \in E$, we have

$$
\begin{aligned}
\left|\left(B_{i} y\right)\left(n_{1}\right)-\left(B_{i} y\right)\left(n_{2}\right)\right| & \leq \sum_{m \in \mathbb{Z}}\left|a_{i}\left(n_{1}, m\right)-a_{i}\left(n_{2}, m\right)\right| \cdot\left|g_{i}(m, y(m))\right| \\
& \leq\left\|\tilde{a}_{i}\left(n_{1}\right)-\tilde{a}_{i}\left(n_{2}\right)\right\|_{q} \cdot\left\|\mu_{i}^{M}\right\|_{p},
\end{aligned}
$$

which yields that each $B_{i}(E)$ is equi-asymptotically almost periodic since $\widetilde{a}_{i} \in A A P\left(\mathbb{Z}, l^{q}(\mathbb{Z})\right)$. Then, by Theorem 2.3, each $B_{i}(E)$ is precompact in $A A P(\mathbb{Z})$. Let $\left\{y_{k}\right\} \subset E$. Then $\left\{B_{i} y_{k}\right\}$, if necessary going to a subsequence, is convergent in $A A P(\mathbb{Z})$ for each $i \in\{1,2, \ldots, \lambda\}$. By (3.1), we conclude that $\left\{\mathcal{N} y_{k}\right\}$ is convergent in $A A P(\mathbb{Z})$. So, $\mathcal{N}(E)$ is precompact in $A A P(\mathbb{Z})$.

By applying Schauder's fixed point theorem, there exists a fixed point $y *$ of $\mathcal{N}$ in $E$. Then we have

$$
y_{*}=\mathcal{N} y_{*}=x_{y^{*}}=\sum_{i=1}^{\lambda} A_{i} x_{y^{*}} \cdot B_{i} y_{*}=\sum_{i=1}^{\lambda} A_{i} y_{*} \cdot B_{i} y_{*}=\mathcal{M} y_{*},
$$

which means that $y_{*}$ is a fixed point of $\mathcal{M}$. This completes the proof. 
Finally, we give a simple example to illustrate our result.

Example 3.2 Let $\lambda=2, p=1, q=\infty$,

$$
\begin{aligned}
& f_{1}(n, x)=\frac{x}{10}\left(\sin n+\sin \pi n+\frac{1}{|n|+1}\right), \quad g_{1}(n, x)=\frac{\sin \left(x e^{n^{2}}\right)}{2\left(1+n^{2}\right)}, \\
& a_{1}(n, m)=\frac{\cos n+\cos \sqrt{2} n+\frac{1}{n^{2}+1}}{3\left(1+m^{2}\right)},
\end{aligned}
$$

and

$$
f_{2}(n, x)=\frac{\cos n \sin x}{20}, \quad g_{2}(n, x)=\frac{\arctan (n x)}{1+n^{2}}, \quad a_{2}(n, m)=\frac{1}{3} e^{-m^{2}} \sin n .
$$

It is easy to see that (H1) holds with $L_{1}=\frac{3}{10}$ and $L_{2}=\frac{1}{20}$. Also, (H2) holds with $\mu_{1}^{r}(n) \equiv$ $\frac{1}{2\left(1+n^{2}\right)}$ and $\mu_{2}^{r}(n) \equiv \frac{\pi}{2} \cdot \frac{1}{1+n^{2}}$. In addition, (H3) can be easily verified. By a direct calculation, we can get

$$
\alpha_{1} \leq 1, \quad \alpha_{2} \leq \frac{1}{3}
$$

and

$$
\left\|\mu_{1}^{r}\right\|_{1} \leq \frac{\pi+1}{2}, \quad\left\|\mu_{2}^{r}\right\|_{1} \leq \frac{\pi^{2}+\pi}{2}, \quad \forall r>0 .
$$

Letting $M=1$, we have

$$
\sum_{i=1}^{2} \alpha_{i} L_{i}\left\|\mu_{i}^{M}\right\|_{1} \leq \frac{3(\pi+1)}{20}+\frac{\pi^{2}+\pi}{120}<1,
$$

and

$$
\sum_{i=1}^{2}\left[\sup _{n \in \mathbb{Z},|x| \leq K}\left|f_{i}(n, x)\right| \cdot \alpha_{i} \cdot\left\|\mu_{i}^{M}\right\|_{1}\right] \leq \frac{3(\pi+1)}{20} K+\frac{\pi^{2}+\pi}{120}<K, \quad \forall K>1 .
$$

Thus, (H4) holds with $M=1$. Then, by using Theorem 3.1, Equation (1.1) has an asymptotically almost periodic solution.

Competing interests

The authors declare that they have no competing interests.

\section{Authors' contributions}

WL completed the main study, carried out the results of this article and drafted the manuscript. WP checked the proofs and verified the calculation. All the authors read and approved the final manuscript.

\section{Acknowledgements}

The work was supported by the NSF of China (11101192), the Key Project of Chinese Ministry of Education (211090), the NSF of Jiangxi Province, the Foundation of Jiangxi Provincial Education Department (GJJ12205), and the Research Project of Jiangxi Normal University (2012-114). 


\section{References}

1. Agarwal, RP: Difference Equations and Inequalities. Theory, Methods, and Applications, 2nd edn. Monographs and Textbooks in Pure and Applied Mathematics. Marcel Dekker, New York (2000)

2. Diblík, J, Schmeidel, E, Ružičková, M: Existence of asymptotically periodic solutions of system of Volterra difference equations. J. Differ. Equ. Appl. 15, 1165-1177 (2009)

3. Diblík, J, Ružičková, M, Schmeidel, E: Existence of asymptotically periodic solutions of scalar Volterra difference equations. Tatra Mt. Math. Publ. 43, 51-61 (2009)

4. Diblík, J, Schmeidel, E, Ružičková, M: Asymptotically periodic solutions of Volterra system of difference equations. Comput. Math. Appl. 59, 2854-2867 (2010)

5. Diblík, J, Ružičková, M, Schmeidel, E, Zbaszyniak, M: Weighted asymptotically periodic solutions of linear Volterra difference equations. Abstr. Appl. Anal. 2011, Article ID 370982 (2011). doi:10.1155/2011/370982

6. Diblík, J, Schmeidel, E: On the existence of solutions of linear Volterra difference equations asymptotically equivalent to a given sequence. Appl. Math. Comput. 218, 9310-9320 (2012)

7. Ding, HS, Chen, YY, N'Guérékata, GM: $C^{n}$-almost periodic and almost periodic solutions for some nonlinear integral equations. Electron. J. Qual. Theory Differ. Equ. 6, 1-13 (2012)

8. Araya, D, Castro, R, Lizama, C: Almost automorphic solutions of difference equations. Adv. Differ. Equ. 2009, Article ID $591380(2009)$

9. Blot, J, Pennequin, D: Existence and structure results on almost periodic solutions of difference equations. J. Differ Equ. Appl. 7, 383-402 (2001)

10. Cuevas, C, Henríquez, H, Lizama, C: On the existence of almost automorphic solutions of Volterra difference equations. J. Differ. Equ. Appl. (in press)

11. Ding, HS, Fu, JD, N'Guérékata, GM: Positive almost periodic type solutions to a class of nonlinear difference equations. Electron. J. Qual. Theory Differ. Equ. 25, 1-16 (2011)

12. Hamaya, Y: Existence of an almost periodic solution in a difference equation with infinite delay. J. Differ. Equ. Appl. 9 227-237 (2003)

13. Pennequin, D: Existence of almost periodic solutions of discrete time equations. Discrete Contin. Dyn. Syst. 7, 51-60 (2001)

14. Corduneanu, C: Almost Periodic Functions, 2nd edn. Chelsea, New York (1989)

15. Fink, AM: Almost Periodic Differential Equations. Lecture Notes in Mathematics. Springer, Berlin (1974)

16. Zhang, C: Almost Periodic Type Functions and Ergodicity. Kluwer Academic, Dordrecht (2003)

doi:10.1186/1687-1847-2012-199

Cite this article as: Long and Pan: Asymptotically almost periodic solution to a class of Volterra difference equations. Advances in Difference Equations 2012 2012:199.

\section{Submit your manuscript to a SpringerOpen ${ }^{\circ}$ journal and benefit from:}

- Convenient online submission

- Rigorous peer review

Immediate publication on acceptance

- Open access: articles freely available online

- High visibility within the field

- Retaining the copyright to your article 